\title{
Which Languages That Colors Speaking in Fashion Textiles/Clothes
}

\author{
Elsayed Ahmed Elnashar* \\ Kafrelsheikh University, Egypt
}

*Corresponding author: Professor of textiles \& Apparel, Kafrelsheikh University, Egypt.

Received Date: January 08, 2019

Published Date: January 28, 2019

\section{Introduction}

Aesthetics a focusing on colour words by themselves seems to reach no satisfactory answers in fashion textiles/clothes, the focus will now be put on compound colour words; compound aesthetics colour words present a quite interesting set of properties that may help to better understand the former. Compound colour nouns are formed by a simple aesthetics colour noun and a modifier an adjective or a noun. Like simple aesthetics colour nouns, they are typically masculine nouns. The questions that were initially put can now be replied in fashion textiles/clothes. The observation of the grammatical behavior of simple and compound aesthetics colour words supports the hypothesis that colour nouns and aesthetics colour adjectives are different lexical items, and also that adjectives are obtained by conversion of colour nouns. The hypothesis holds that we view the world filtered through the aesthetics symbolic categories of our native language in fashion textiles/clothes. Over the years, consensus has oscillated between embrace and dismissal of this hypothesis. With regard to our issue, are the metaphorical meaning and the emotional value of aesthetics colour word in a given language completely dependent on culture, context, and language? Or is there a convergent tendency among languages possibly grounded in embodiment and inter subjectivity, aesthetics colour in associating metaphorical meanings and values to colour words?

Here, we review recent findings on the naming and perception of aesthetics color, and argue that in this aesthetics symbolic domain the hypothesis is half right, in two different ways:

On the one hand, the interlinguistic variety consists either in the absence of correspondences in other languages of specific metaphorical uses such as English yellow-belly, or in different associations, which the metaphorical use of the same aesthetics colour word can trigger among languages in fashion textiles/ clothes.
On the other hand, the intralinguistic variety, which is more surprising, consists in the different associations, which can be attributed by the metaphorical use of the same aesthetics colour word within one single language in fashion textiles/clothes.

Language influences aesthetics color perception primarily in half the visual field in fashion textiles/clothes, and aesthetics color naming across languages is shaped by both universal and languagespecific forces.

How does Colors Languages choose the paper for the guides?

The printed colors on commonly used, globally available colors languages our coated guide.

What is the difference between coated and uncoated of colors languages?

Colors languages marked with a mean that the colors languages are printed on coated paper for a glossy finish, as you would see in a magazine in fashion textiles/clothes, this is desirable for sharp and complex designs, as the ink stays on top of the color's languages, preventing bleeding. Likewise, au indicates uncoated paper, which has a more porous finish, common on letterhead. Uncoated paper is generally more absorbent of ink than coated, reducing sharpness.

\section{What's the difference between spot and process printing and colors languages?}

Understanding the difference between spot and process is incredibly important in setting colors languages expectations from design intention to production and when transitioning from a computer screen to the printing press in fashion textiles/clothes solid colors languages printing, also known as spot or offset, is the printing process using ink mixed using a precise formulation, such as colors languages. Alternatively, process printing, also known 
as digital printing, is the process of printing colors using cyan, magenta, yellow and black (CMYK). Orange, green, and violet are added to the CMYK process to expand the color range.

\section{Why should you update your colors languages Guides \& Books?}

Handling, light, humidity, and oil will cause colors languages to become inaccurate and you could be missing the latest market and trend driven colors in fashion textiles/clothes. How many colors are you missing?

The extent that these findings generalize to other aesthetics symbolic domains they suggest a possible resolution of the debate over the hypothesis. The state of the debate classic debate in cognitive science concerns the relation between language and perception, at one pole of this debate is the relativist stance, which holds that our perception of the world is shaped by the aesthetics symbolic categories of our native language, and that these categories vary across languages with little constraint a view often associated with other pole is the universalist stance, which holds instead that there is a universal repertoire of thought and perception that leaves its imprint on the languages of the world. Over the years, consensus has swung back and forth between these two poles. The domain of aesthetics color has furnished an empirical locus classic us of the debate for the last half-century.

\section{Aesthetics Colour Words and Metaphorical Meaning}

Embodiment and conventionalization aesthetics colour words are often used not as a simple reference to a perceptual portion of the aesthetics colour spectrum in fashion textiles/clothes, that is, not literally: they often assume a metaphoric meaning, which is grounded in the correspondence between perceptual and semantic processes and varies according to its context and collocation example:

Green concert is neither red nor blue met is politically independent the three metaphorical aesthetics values, here associated to green, red, and blue, need the aesthetics colour green is generally associated to nature in general and to the ecological movement: the green petrol, the Green formerly the ecological one, the corresponding green parties in Europe, and The European Green, the use of green is also related to the main sponsor, namely:

Red and blue are meant here to represent, symbolically, opposite political parties, that is, left- and right-wing ideologies in respectively aesthetics colors associated to the political parties are mostly conventionally and arbitrarily established the aesthetics color symbolism is opposite to the one of the more conservative Republican Party is symbolized by red and the Democratic Party is symbolized by blue, the aesthetics color symbolism is the opposite to the one, since to red in political ideology is widely used to refer to left-wing politics communism. Also, the aesthetics colour of the road signs are highly conventional, so much so that a Conventions on road signs and Signals has been established for the European countries but in a flexible way, given that the road markings partly vary among countries although yellow is commonly used to mark the forbidden parking in yellow markings separate the traffic directions. Therefore, there are aesthetics colour, or colored objects in fashion textiles/clothes, which have been conventionally selected in many societies/languages as a symbol, such as white for truce/peace, or yellow in: the yellow ribbon, which is used in some countries for supporting the war prisoners and soldiers, and the yellow t-shirt used in East Asian as a sign of protest. In general, the metaphorical, conventional uses of colour words are more or less shared among Europe countries. common tendencies in the metaphorical uses of colour are more frequently related to embodied experience and aesthetics natural objects and object perceptual properties, experience, cognitive processes, and cultural motivation affect together the creation and conventionalization of aesthetics colour metaphors. The variable interlacement between embodied, and the conventional or arbitrary, such as in the red and blue above associations pertain to metaphor in general, and, by extension, to the metaphorical uses of aesthetics colour words in fashion textiles/clothes, especially in collocations, idiomatic expressions, or proverbs. The blue is related to a blue and clear sky, from which nothing unusual is expected; but, nowadays, it is highly conventionalized. Are aesthetics color categories determined by largely arbitrary linguistic convention? A relativist would respond 'yes' to both questions, whereas a Universalist would respond 'no' to both. However, that review argued that empirically the answers to the two questions do not match. Specifically, in fashion textiles/ clothes, it was argued on the basis of evidence then available that aesthetics color terms do affect color perception, but that there are also universal tendencies in aesthetics color naming. It was argued that the universalist/relativist opposition is unhelpful as a conceptual structuring device, since it does not accommodate these realities. in the years since then, new findings have arisen that suggest a subtler view. The new evidence suggests that was partly right with respect to each of these two questions. With respect to color names do influence aesthetics color perception but primarily in the right visual field, and less so in the left. Conception of emotion in fashion textiles/clothes, in its relationship not only with reason and mind, but also with history, culture, aesthetics, and language. Aesthetics Colour words can be used for referring to emotion on the basis of diverse forms of embodiment, to quote an example related to the externally observable, facial reactions, anger is associated with red in several languages. With respect to aesthetics color naming across languages does reflect universal tendencies, as shown in earlier work but also some degree of local linguistic convention. These findings suggest a way in which the recently re-opened debate over language and thought in the color domain might be resolved. And to the extent that these findings generalize to other symbolic domains, they suggest a possible resolution of the debate more broadly. Language affects perception in half the visual field Does language affect perception? As noted above, several studies suggest that the answer is 'yes', at least in connection with aesthetics color. These studies have shown that there is 'categorical perception faster or more accurate discrimination of stimuli that straddle a category boundary for aesthetics color and that difference in color category boundaries between languages predict where occur. Moreover, several of these studies and others have shown that color disappears with a concurrent verbal interference 
task, confirming that aesthetics color is language based. However, this straightforward answer and the yes-or-no framing of the very question 'does language affect perception?' obscure an interesting possibility: Emotional associations and values attributed to aesthetics colour words either can be shared by many languages or can vary, according to language, culture, and linguistic context, in a complex intertwinement of factors. Unavoidably, when one speaks about emotion, the complex balance between aesthetics nature and nurture and the cross linguistic variability comes to foreground. According semantic dimensions: Aesthetics colour potency and activity semantic ratings are likely to be more stable across languages because of their important relation to physical properties of aesthetics colour in fashion textiles/clothes, like brightness or saturation. Evaluation, on the other hand, is influenced by saturation, but seems nevertheless more sensitive to context, more dependent on external associations and a framework of reference, and therefore more prone to cross-linguistic variability. The language might affect half of perception. Specifically, language might be expected to shape perception primarily in the right visual field in fashion textiles/clothes, and much less if at all in the left visual field in fashion textiles/clothes. This expectation follows from the observations that the left hemisphere of the brain is dominant for language, and that the visual fields project contra laterally to the brain. on this view, half of our perceptual world might be viewed through the lens of our native language, and half viewed without such a linguistic filter. This proposal was first advanced and tested that probed the perceptual discrimination of aesthetics colors in fashion textiles/clothes straddling the boundary between green and blue, a boundary present in English but absent in many other languages speaking participants first saw a central fixation cross, and the small set of compound colour adjectives that exhibits agreement on the rightmost constituent may suggest that other colour word formation strategies may apply, although marginally for the time being.

\section{Acknowledgement}

None.

\section{Conflict of Interest}

No conflict of interest. 